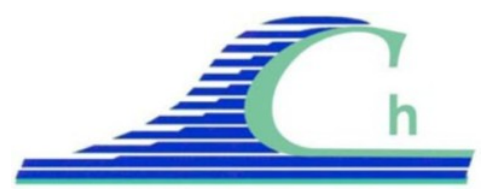

XII ${ }^{\text {ìmes }}$ Journées Nationales Génie Côtier - Génie Civil

Cherbourg, 12-14 juin 2012

DOI:10.5150/jngcgc.2012.092-B @ Editions Paralia CFL

disponible en ligne - http://www.paralia.fr - available online

\title{
Comprendre le transport sédimentaire des franges littorales en rive sud d'un estuaire macrotidal tempéré : la baie de Somme, Picardie, France
}

\author{
Julia BASTIDE ${ }^{1}$, Edward ANTHONY ${ }^{2}$, Franck DOLIQUE ${ }^{3}$
}

1. Laboratoire d’Océanologie et de Géoscience - UMR LOG 8187, 28 avenue Foch, BP 8062930 Wimereux, France.

julia.bastide@orange.fr

2. C E R E G E : Centre Européen de Recherche et d'Enseignement des Géosciences de l'Environnement. anthony@cerege.fr

3. UNIVERSITE ANTILLES-GUYANE Campus de Martinique BP 7207, 97275 Schoelcher Cedex, Martinique - FWI.

franck.dolique@martinique.univ-ag.fr

\section{Résumé :}

La baie de Somme est l'archétype d'un estuaire macrotidal tempéré fortement anthropisé et présentant des enjeux divers et parfois conflictuels en termes d'aménagement et d'écologie. Caractérisé par un marnage mégatidal à l'embouchure (>8 m par vive-eau), l'estuaire est, par excellence, un attracteur de sédiments, avec un delta de comblement important alimenté en rive sud par des sables marins et des galets en provenance d'un couloir de transit qui longe une partie de la côte française de la Manche. Différentes techniques complémentaires de mesures in situ, en rive sud de cet estuaire ont permis d'apprécier la direction et l'intensité du transport des sédiments. L'objectif étant de comprendre les vitesses de transport des matériaux contribuant à la dynamique évolutive de cette rive.

Mots clés :

Morphodynamique littorale - Aménagement littoral - Estuaire macrotidal - Baie de Somme

\section{Introduction}

Les estuaires actuels sont des entités géologiques jeunes qui se comblent à des rythmes divers. Ce comblement est assuré par la conjonction d'une décharge fluviale et d'eau de mer dont la convergence conduit à un piégeage aussi bien de la charge de fond que des sédiments en suspension dans la partie basse des embouchures fluviales envahies par la mer. Ce comblement peut être rapide, mais peut aussi être extrêmement lent (PETHICK, 1996) en fonction du contexte géomorphologique, hydrodynamique, sédimentaire et eustatique. À ces paramètres, il faut ajouter l'influence de l'homme qui peut affecter le rythme de remplissage. L'estuaire de la Somme conjugue plusieurs 
éléments qui ont favorisé son comblement, sauf en ce qui concerne les apports de sédiments fluviaux qui restent tout à fait modiques. Cette modicité de la décharge solide fluviale implique donc un remplissage essentiellement d'origine marine. Mais elle implique aussi que cet estuaire risque peu d'évoluer vers ce stade ultime des embouchures fluviales qu'est le delta, pourvoyeur lui, de sédiments à la mer. Cet article propose de faire la synthèse et de discuter des modalités du transit sédimentaire en rive sud de l'estuaire.

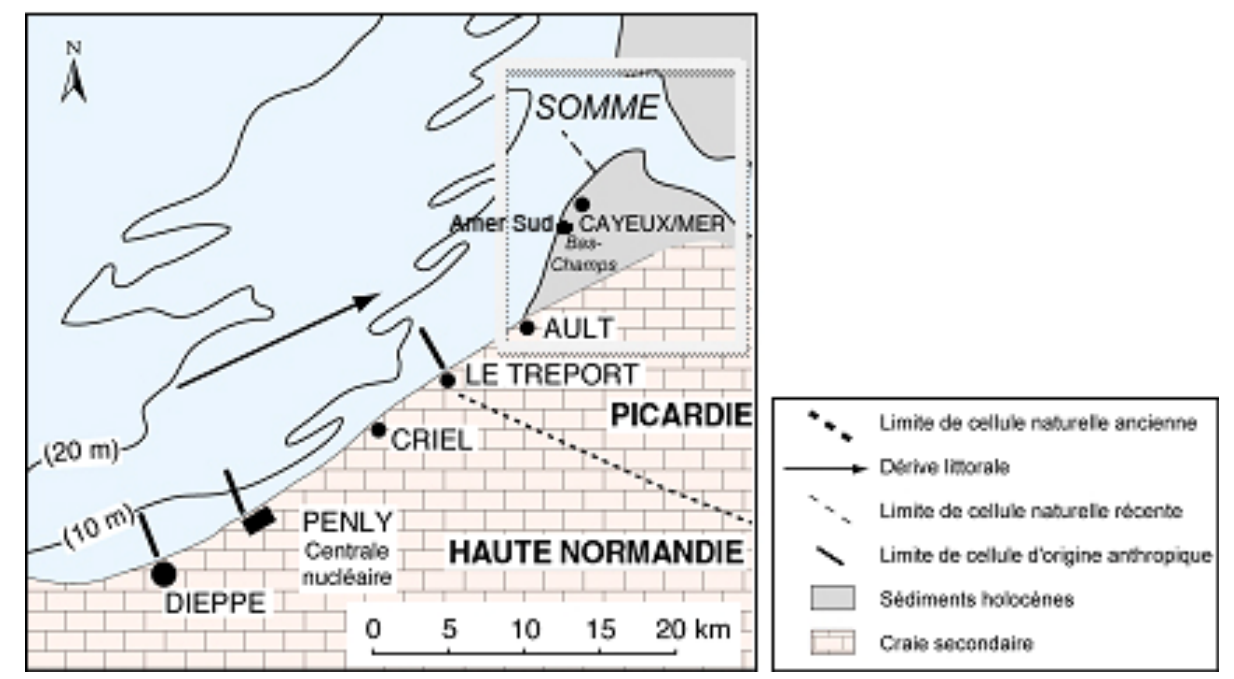

Figure 1. Localisation du site d'étude, rive sud de l'estuaire de la Somme.

\section{Matériel et méthodes}

La démarche méthodologique adoptée dans cet article vise à décrire les vitesses de transports des matériaux de la frange sud de l'estuaire de la Somme. Celui-ci est fortement perturbé par le tarissement progressif des apports sédimentaires naturels dans la mesure où il s'agit d'un cordon de galets littoral ayant un «alignement de dérive ». Pour répondre à cette problématique, la démarche méthodologique porte sur le montage de campagnes d'expérimentation de terrain, localisées au niveau du dernier épi, à l'amer Sud de Cayeux-sur-Mer (figure 1 et 2), site régulièrement rechargé en galets de carrière. La figure 2 montre, respectivement la zone rechargée en galets en début de campagne expérimentale, et un modèle numérique de terrain indiquant le point de déploiement des galets de traçage.

Le sommet de la berme de galets avec une élévation de 10 à 12 m NGF (figure 2) correspond au rechargement réalisé avant l'expérimentation. Le profil est pentu avec une descente à la mer s'accentuant vers le bas. Ces conditions de réflexion de la digue de galets ne permettent pas une absorption de l'énergie de la houle mais créent une marche d'attaque et de source d'alimentation de la dérive littorale du fait d'un swash très actif. 


\section{XII ${ }^{\text {èmes }}$ Journées Nationales Génie Côtier - Génie Civil \\ Cherbourg, 12-14 juin 2012}
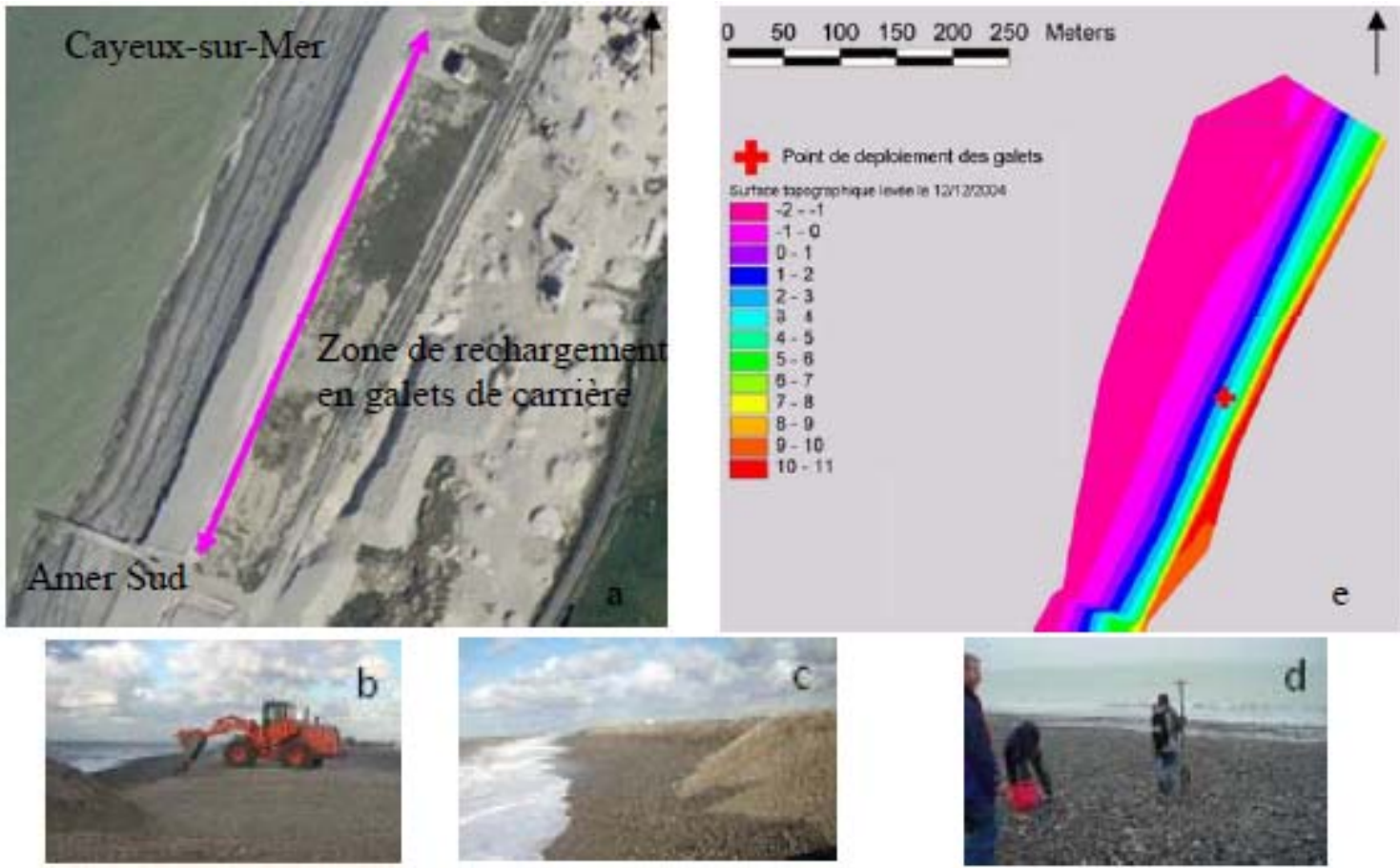

Figure 2 (a) Zone d'expérimentation. (b) Rechargement artificiel du cordon de galets de l'amer Sud. (c) Cordon artificiel succédant le rechargement. (d) Photographie du levée au DGPS. (e) MNT de l'état initial du site de déploiement des galets.

\subsection{Approche expérimentale}

Essayer de comprendre la morphodynamique en rive sud de la Somme passe par un couplage étroit entre des approches morphométriques et hydrodynamiques. Pour ce faire, des campagnes de terrain ont été réalisées sur une période de temps allant de 3 à 15 jours. Cette durée brève des campagnes était dictée par la nécessité d’un compromis entre des relevés de données suffisamment longs pour permettre une caractérisation morphodynamique englobant différentes situations hydrodynamiques (temps calme, temps perturbé, tempêtes, cycle de marée semi-mensuel comportant vive-eau et morteeau) et des déploiements de matériels, dans un site exposé à des dégradations potentielles du fait de la proximité de nombreuses activités de loisirs pratiquées sur les plages péri-estuariennes et la baie de Somme.

\subsection{Les techniques de quantifications des déplacements des sédiments}

De nombreuses approches, directes et indirectes permettent de mesurer les taux de transports (LEVOY \& MONFORT, 2002). Pour être utilisable comme marqueur, le matériel adopté doit avoir un comportement dynamique aussi proche que possible de celui du stock sédimentaire dont les déplacements sont étudiés. La quantification des déplacements est fonction des sites d'injection. 


\subsubsection{Les sables fluorescents à la base du cordon}

Cette technique a été utilisée lors d'études portant sur le transport sédimentaire sur des plages à barres intertidales de la Côte d'Opale (CORBAU, 1995 ; LEVOY et al., 1998). La technique est fastidieuse et ne donne pas nécessairement des résultats probants ou faciles à quantifier. Nous l'avons utilisée lors de deux campagnes en décembre 2004 et 2005. Le sable, après avoir été prélevé sur le site, lavé et séché, a été recouvert de peinture fluorescente à l'aide d'une bétonnière. Après une nouvelle période de séchage permettant la fixation de la peinture sur les grains, le traceur a été injecté sur site à marée basse dans le but de faire une série de prélèvements autour du point d'injection le lendemain matin afin d'établir le nuage de dispersion induit par l'action d'une marée. Quinze kilogrammes de traceur ont été injectés sur un carré de $50 \mathrm{~cm}$ de côté et recouvert d'une pellicule d'un centimètre de sable non fluorescent.

\subsubsection{Les galets en résine synthétique pour définir la mobilité des galets et du cordon}

Pour le suivi sédimentaire en rive sud de la baie de Somme deux techniques ont été mises en place pour connaître les capacités et les taux de dispersion des galets :

- i) les galets extraits du site, marqués au feutre et enduits de peinture fluorescente (figure 3a),

- ii) les galets en résine synthétique fabriqués par l'université de Sussex (figure 3 b).

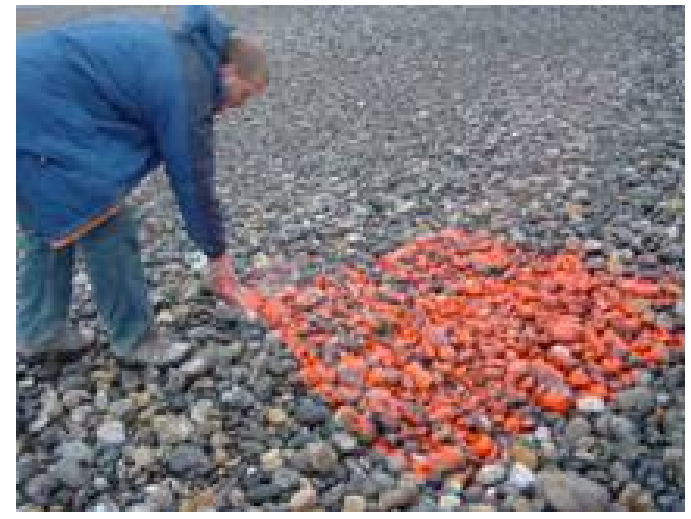

Figure 3a. Déploiement des galets peints.

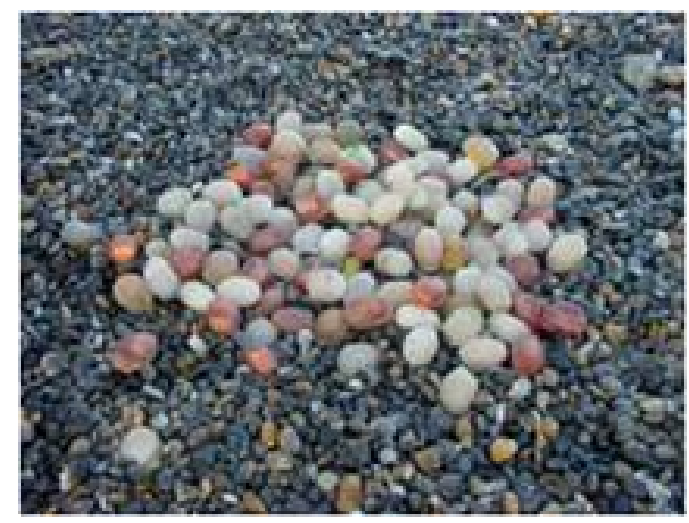

Figure 3b. Déploiement sur site des galets en résine.

Les galets synthétiques résultent de la recherche en collaboration dans le cadre projet Interreg III, PAR/BAR. Ces galets synthétiques en résine ont été confectionnés avec en leur centre une pièce de cuivre afin de faciliter leur localisation au détecteur de métaux après dispersion par la marée. Les galets déployés ont un calibre de $4.5 \mathrm{~cm}$ en largeur et 6 à $7 \mathrm{~cm}$ de longueur. Au total 220 galets on été injectés à 2 reprises.

- Du 13/12/2004 au 18/12/2004,

- Du 28/10//2005 au 11/11/2005. 


\section{XII ${ }^{\text {èmes }}$ Journées Nationales Génie Côtier - Génie Civil \\ Cherbourg, 12-14 juin 2012}

La mesure de la dispersion s'est faite pour deux classes granulométriques de sédiments. Chaque galet porte un numéro qu'il a fallu noter dans le GPS afin de pouvoir estimer les pertes sur l'échantillon d'origine. Pour caler le modèle numérique de terrain de la dispersion des galets et surtout établir la force du transit, les courantomètres de type S4ADW ont été déployés sur la zone de travail.

Le taux de galets peints retrouvés correspond à moins de $5 \%$ de la fraction déposés sur site ; c'est pourquoi cette méthode a été rapidement abandonnée pour que celle des galets synthétiques. Ce choix a été dicté par un hydrodynamisme local important, permettant un remaniement de la surface de la plage sur plus de $10 \mathrm{~cm}$ en profondeur. Ainsi, à l'œil nu il était quasi impossible de détecter les éléments disposés alors que le détecteur de métaux le permettait. À chaque passage du détecteur de métaux, un modèle numérique de terrain a été établi afin de connaitre les variations morphologiques de la plage.

\section{Résultats}

Les traceurs, utilisés in situ, donnent une image de la configuration des transports qui s'effectuent à proximité immédiate des fonds. Cette approche permet de réaliser des estimations qui sont le reflet de l'action combinée des agents hydrodynamiques et matériels du site (figure 4 exemple sur l'expérimentation du 13/12/2004 au 18/12/2004).
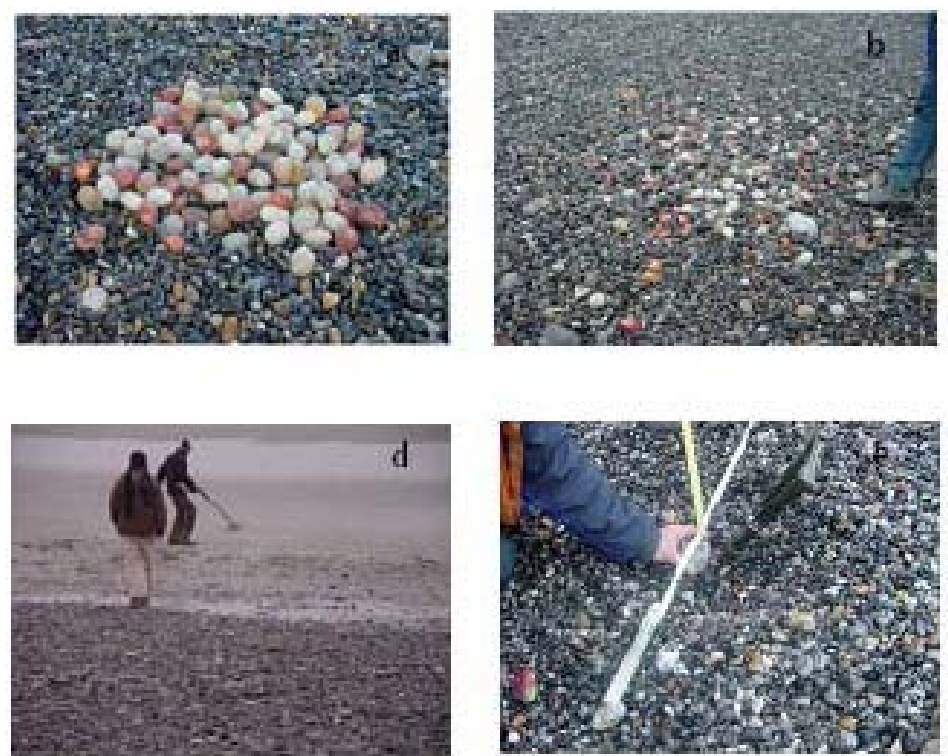

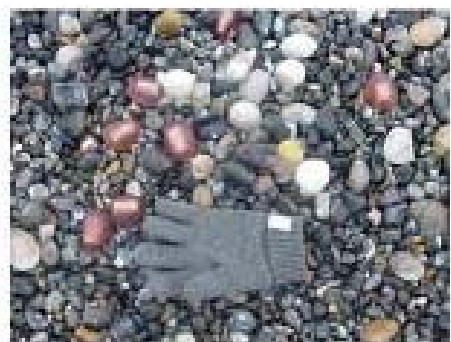

a : dépôt des galets résines,

b : dispersion après un cycle de marée,

c : échelle des galets,

d: détecteur de métaux utilisé pour la recherche des galets résines à mi estran,

e : mesure de l'espacement et de la profondeur de remaniement.

Figure 4. Photographies illustrant les phases successives de mise en place, de dispersion et de recherche des galets de résine sur un cycle de marée. 
Lors du premier jour d'analyse, plus de 85\% des galets en résine ont été retrouvés dans un périmètre très proche, à quelques mètres, de la zone d'injection (figure 5). Au fur et à mesure de la campagne, la dispersion des matériaux a été amplifiée tant en surface de remaniement qu'en orientation, reflétant la transition de conditions de faible agitation vers l'agitation forte associée à la tempête, (figure 5). Les directions de dispersion sont matérialisées par les flèches blanches sur cette figure 5. D’un mode essentiellement transversal (cross-shore), en phase de faible agitation du 13/12/04 au 14/12/04, on passe, lors des conditions de tempête, du 16/12/04 au 17/12/04, à un mode nettement dominé par la composante longitudinale (longshore) qui traduit le transit littoral des galets impulsé par la houle. Le transport transversal semble favoriser aussi un enfouissement plus important qui freine le transit longitudinal. Avec l'agitation, la dispersion longitudinale en surface ou proche de la surface $(-5 \mathrm{~cm})$ domine. La dynamique longitudinale de variation topographique est bien mise en évidence sur le MNT différentiel global (figure 6). Sur la durée de la campagne, un retrait de $+7 \mathrm{~m}$ de la berme de rechargement de galets a été observé à la suite de la tempête du 17/12/04 (figure 6).

On note, de façon générale, un transit longitudinal nettement dominant en milieu et haut de plage. Le suivi montre par exemple figure 8, le 13/12/04 une quasi absence de transit en bas de plage, ce qui peut traduire une concentration de l'énergie sur la partie supérieure du profil à marée haute, comme l'ont souligné LEVOY et al. (2002) sur ces plages à fort marnage.

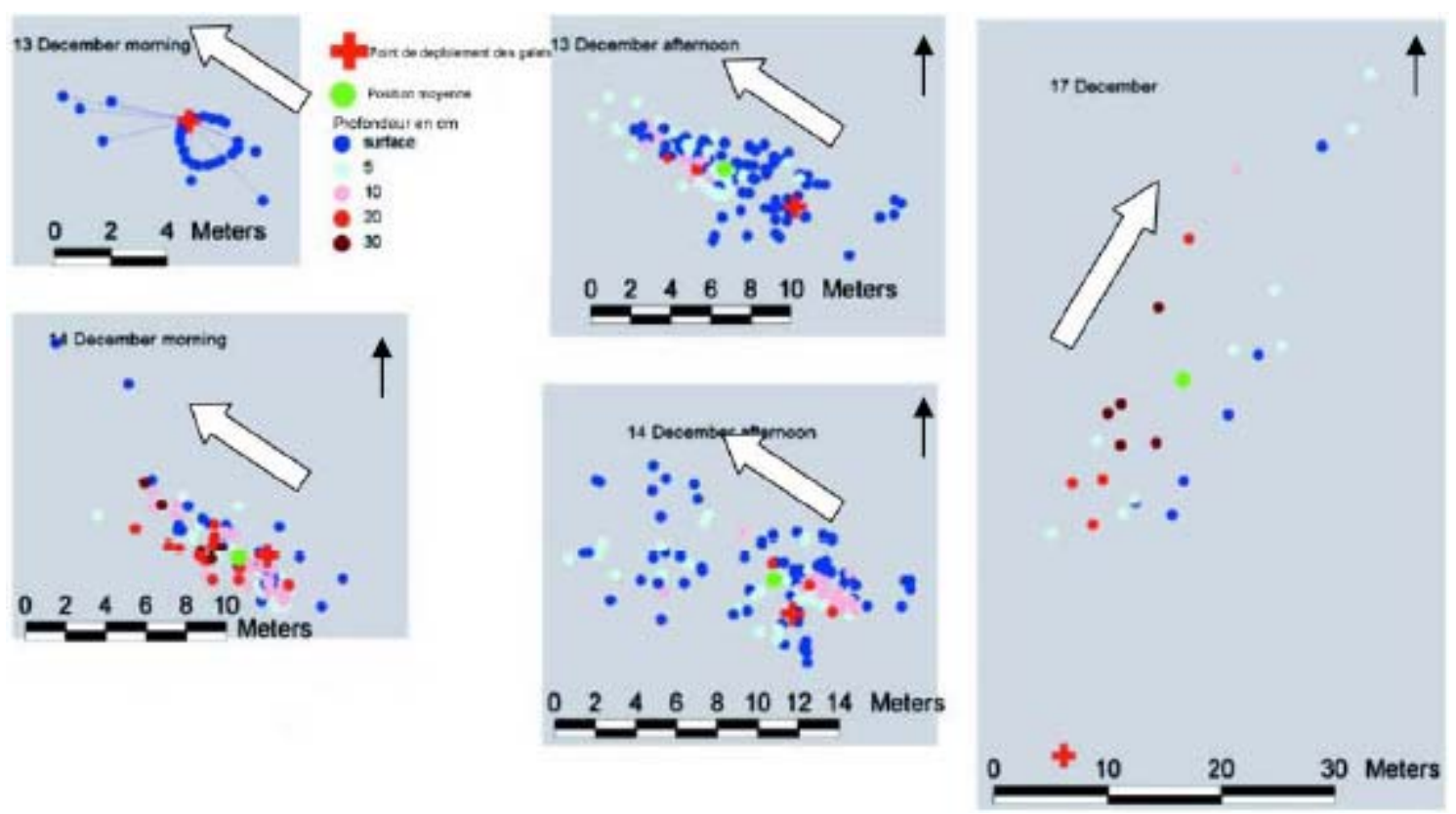

Figure 5. Dispersion des galets en résine entre le 12/12/2004 et 17/12/2004. 


\section{XII ${ }^{\text {èmes }}$ Journées Nationales Génie Côtier - Génie Civil \\ Cherbourg, 12-14 juin 2012}

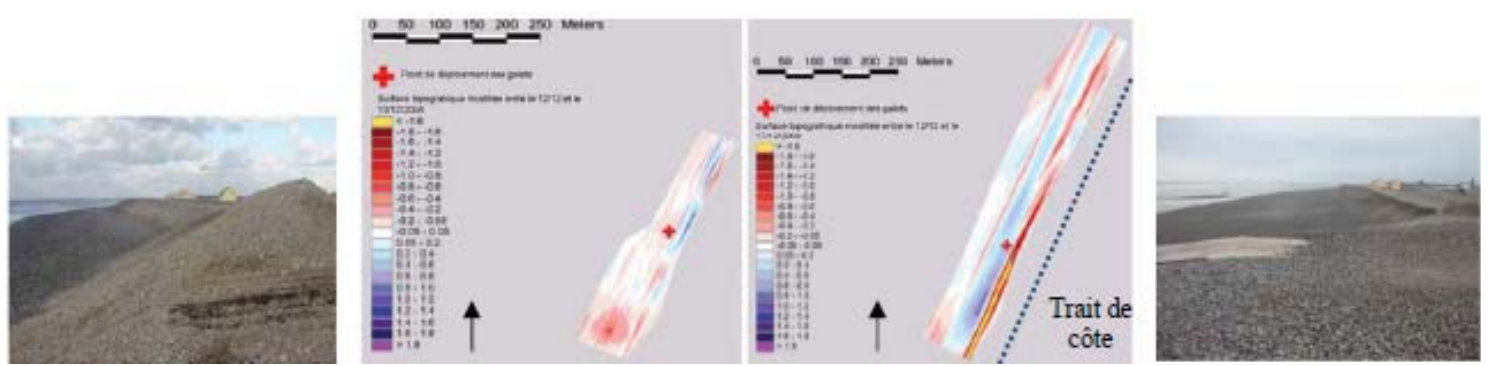

Figure 6. Modèles numériques de terrain du site après le rechargement (gauche) au

12/12/2004 et après le passage de la tempête (droite) au 17/12/2004. On note une érosion de plus de $7 \mathrm{~m}$ de la digue de rechargement en galets avec une dispersion de la plateforme et des bermes successives en formation. Ces changements reflètent un rééquilibrage morphodynamique et sédimentaire du site à la suite de cette intervention anthropique.

Le dernier suivi, du 09/11/05 (figure 7 et 8), montre une plus forte dispersion, avec un transit du bas de plage vers le haut, mais le tout toujours dominé par le transit longitudinal. En ce qui concerne l'enfouissement, un premier constat est que les galets ont tendance à être enterrés sur le site d'injection, avant d'être transportés, ce qui peut traduire l'intensité de l'action des premiers mouvements de swash dans le profil, sur lesquels se surimpose la dérive longitudinale. Dans tous les suivis, les galets les plus proches de la surface sont ceux qui voyagent le plus loin, ceci étant lié à l'exposition de ces galets en surface à des processus de projection lors du déferlement et du swash, mais aussi à une facilité de transport plus grande en surface libre, la forte infiltration de l'eau diminuant l'énergie en profondeur. Les distances moyennes de déplacement des galets par marée à différentes dates sont consignées dans le tableau 1. La distance maximale de transit en un cycle de marée a été de 144.5 m, enregistré le 06/11/05, lors de la période de forte agitation. Dans le cas de strictement la même direction d'approche (SW), la distance moyenne de déplacement des galets, pour des vagues de 0.4 à 0.6 mètres a été de $8,2 \mathrm{~m}$, tandis qu'elle a été de $46,2 \mathrm{~m}$ pour les vagues de $2 \mathrm{~m}$, ce qui démontre la primauté de l'énergie incidente dans le transit. Enfin, il convient de noter que 39\% des galets, soit 220 galets, ont été retrouvés au bout d'une période de 2 semaines. La caractéristique la plus marquante de la zone distale est la présence de crochets marquant la zone terminale du transit des galets. Il s'agit d'un secteur intéressant sur le plan granulométrique, car les crochets de galets reposent sur le plancher sableux (figure 9) qui s'élargit ici à l'entrée de la baie de Somme, tout en créant une zone d'abri derrière permettant une sédimentation de vase, dans les zones les plus abritées. Il existe un gradient d'élévation net du plancher sableux et du contact entre ce dernier et le cordon de galets entre la partie proximale du cordon et cette zone distale en accrétion (figure 9). Ce gradient reflète l'accumulation sableuse croissante vers la baie. Il est connu que la présence d'un plancher sableux a des répercussions 
nettes sur la dynamique du cordon de galets en entraînant d'une part une accélération de la dérive littorale, et en dissipant la houle. Les deux entités ont donc des comportements morphodynamiques très différents qui ont été quantifiés en utilisant divers paramètres.
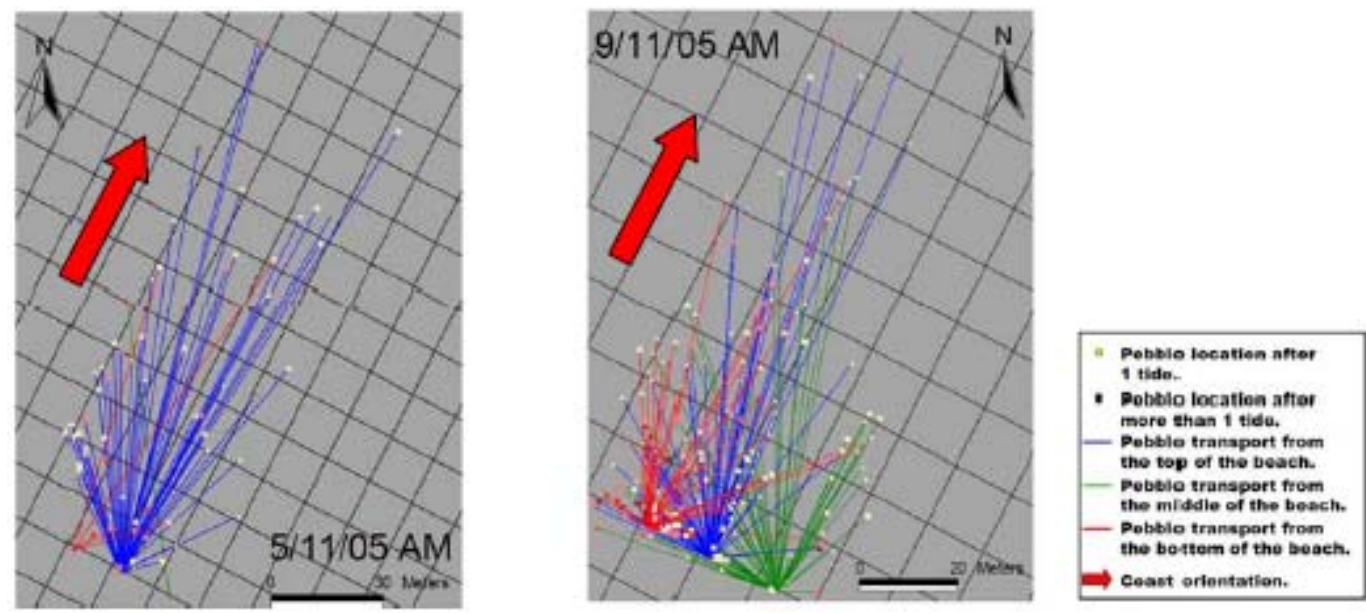

Figure 7. Cartographie de la dispersion des galets sur trois points d'injection : bas de plage, mi-plage et haut-de-plage sur un cycle de marée le 05/11/2005 et 09/11/2005, et la profondeur d'enfouissement des galets.
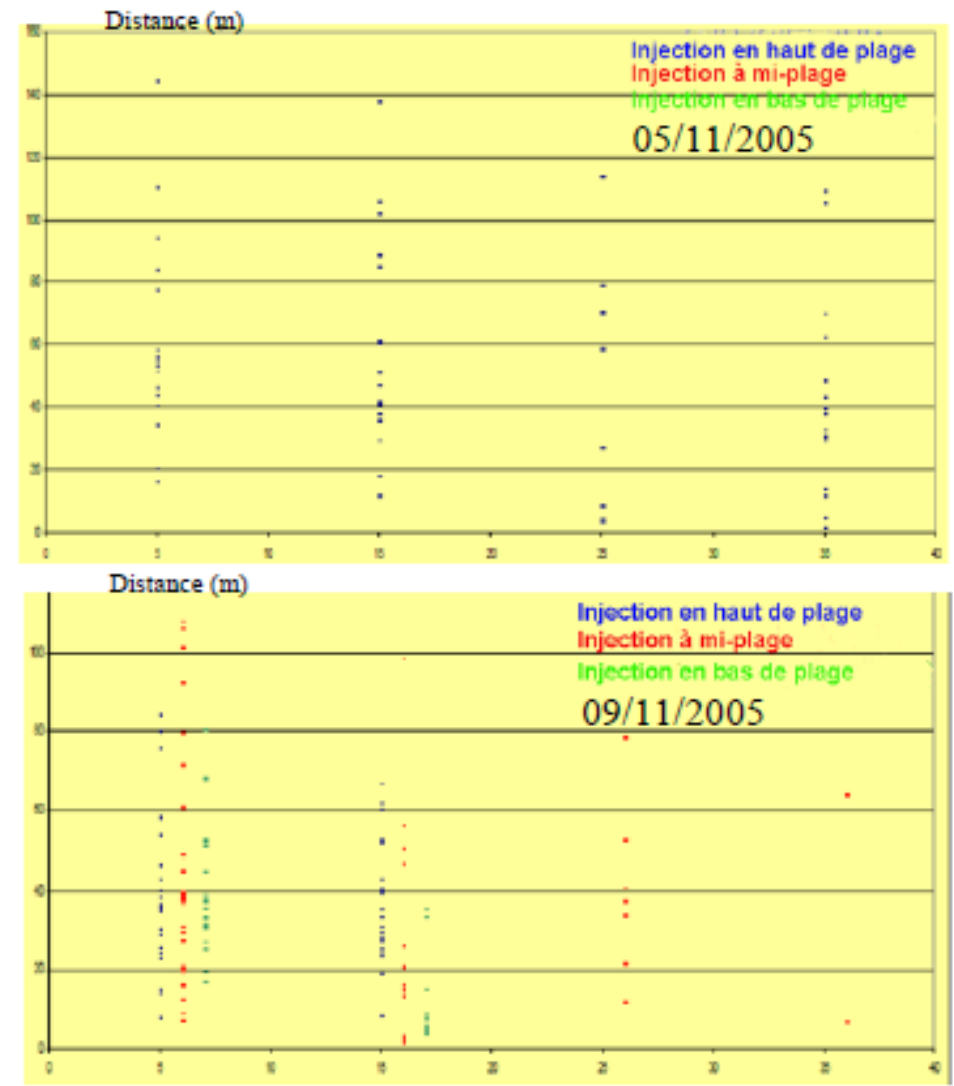

Figure 8. Illustration de la profondeur d'enfouissement des galets. 
Tableau 1. Évaluation de la distance moyenne de transit des galets par marée en milieu de plage.

\begin{tabular}{ll}
\hline Date & $\begin{array}{l}\text { Distance moyenne/marée } \\
\text { (déploiement des galets en milieu de plage) }\end{array}$ \\
\hline $31 / 10 / 2005$ & $8.2 \mathrm{~m} /$ marée \\
$04 / 11 / 2005$ & $46.2 \mathrm{~m} /$ marée \\
$07 / 11 / 2005$ & $44.4 \mathrm{~m} /$ marée \\
\hline
\end{tabular}
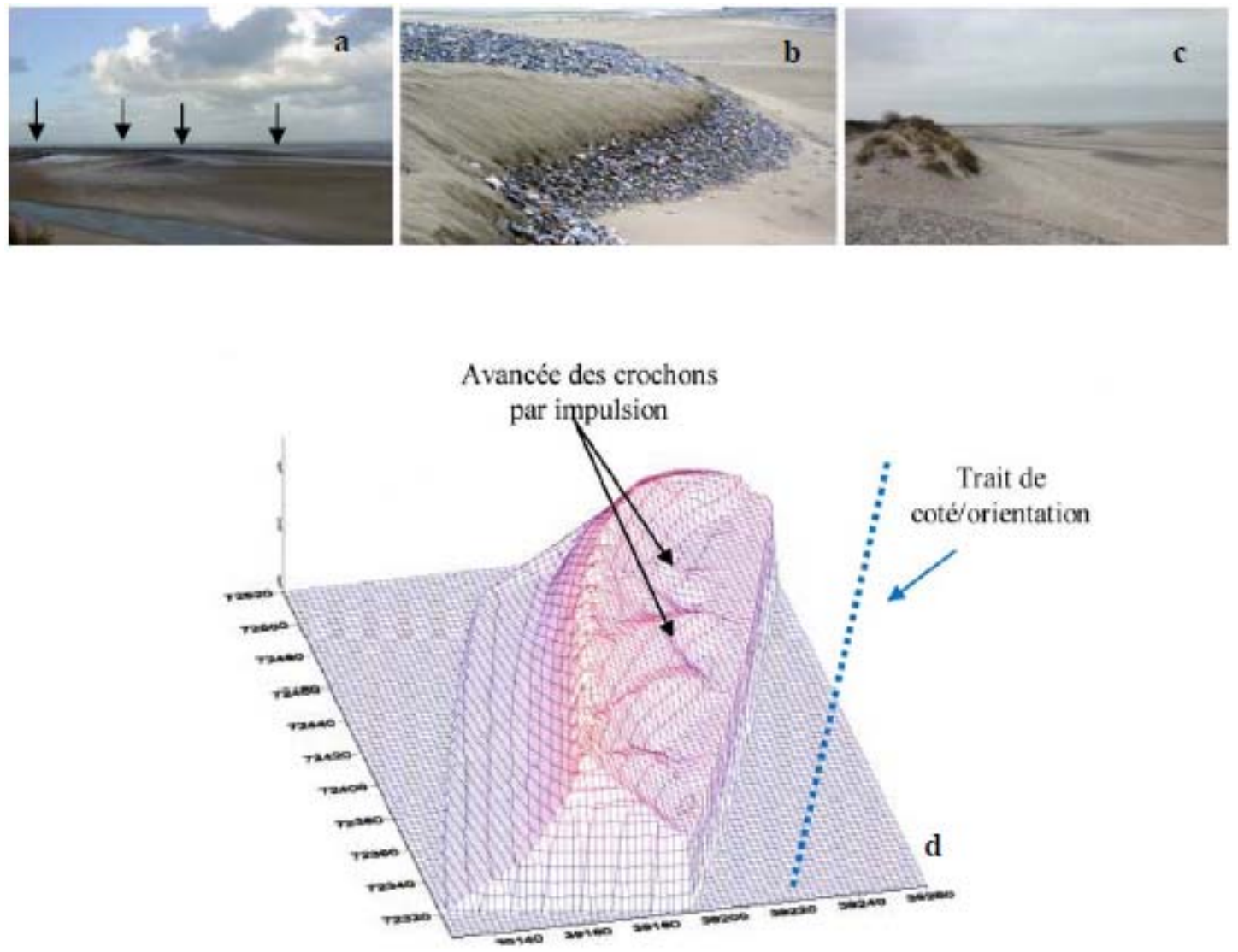

Figure 9. Photographies illustrant la zone terminale du cordon de galets caractérisée par des crochets successifs. $\boldsymbol{a}$ : crochons actifs du cordon de galets entre Cayeux et la

Pointe du Hourdel 2000, $\boldsymbol{b}$ : zoom du cordon, $\boldsymbol{c}$ : état du massif dunaire devant le cordon de galets, $\boldsymbol{d}$ : modélisation de l'avancée des crochons entre la zone proximale et la zone distale.

\section{Conclusion}

Les conditions de transit, dans la zone de rechargement mettent en avant la primauté du transit longitudinal (longshore), en phase avec un cordon à «alignement de dérive ». Plus les conditions d'agitation sont importantes, plus ce transit longitudinal augmente, tandis que les conditions d'agitation faible à modérée sont associées à un mouvement 
transversal (cross-shore), sans que la primauté du transit longitudinal ne soit mise en cause à aucun moment lors de ces conditions plus modérées. Le transit est maximal, pour les galets en surface, l'enfouissement entrainant des taux de déplacement moins importants. L'impulsion au transit des galets en surface est donnée par des projections lors du déferlement et du swash.

\section{Références bibliographiques}

CORBAU C. (1995). Dynamique sédimentaire en domaine macrotidal : exemple du littoral du Nord de la France (Dunkerque). Thèse de doctorat, Université de Lille, Villeneuve-d'Ascq, France, 222 p.

LEVOY F., MONFORT O. (2002). Les transports sédimentaires sur les côtes sableuses, retour d'expérience sur la fiabilité des principales techniques de mesures. VIIème Journées Nationales Génie Civile - Génie Côtier, Anglet, France, 15-16 mai 2002, pp 393-400. doi:10.5150/jngcgc.2002.040-L

LEVOY F., ANTHONY E.J., BARUSSEAU J.P., HOWA H., TESSIER B. (1998). Morphodynamique d'une plage macrotidale à barres. Compte rendu de l'Académie des Sciences de Paris, Sciences de la terre et des planètes, Vol. 327, pp 811-818.

PETHICK J.S. (1996). The geomorphology of mudflats. In: Nordtrom, K.F., Roman, C.T., (eds.), Estuarine Shores - Evolution, Environments and Human Alterations. John Wiley \& Sons, Chichester, pp 185-211. 